PROCEEDINGS OF THE

AMERICAN MATHEMATICAL SOCIETY

Volume 135, Number 1, January 2007, Pages 237-241

S 0002-9939(06)08452-8

Article electronically published on June 30, 2006

\title{
DENSELY ALGEBRAIC BOUNDS FOR THE EXPONENTIAL FUNCTION
}

\author{
SEON-HONG KIM
}

(Communicated by Wen-Ching Winnie Li)

\begin{abstract}
An upper bound for $e^{x}$ that implies the inequality between the arithmetic and geometric means is generalized with the introduction of a new parameter $n$. The new upper bound is smoothly and densely algebraic in $n$, and valid for $-b<x<1$ for arbitrarily large positive $b$ provided that $n(>1)$ is sufficiently close to 1 . The range of its validity for negative $x$ is investigated through the study of a certain family of quadrinomials.
\end{abstract}

\section{INTRODUCTION}

In $\S 4.2$ of the classical treatise [1] the inequality between the arithmetic and geometric means is deduced from

$$
1+x \leq e^{x} .
$$

This is the proof of "Pólya's dream" [5]. With a change of variable this can be rewritten as

$$
e^{x} \leq \frac{1}{1-x}
$$

for $x<1$. In this paper, we shall establish the following generalization of which (1.1) is the case $n=1$. For convenience, we let

$$
U(n, x)=1-\frac{1}{n}+\frac{1}{n}\left(\frac{1+\left(1-\frac{1}{n}\right) x}{1-\frac{x}{n}}\right)^{n} .
$$

Theorem 1.1. For real $n \geq 1$ and

$$
-\frac{n}{n-1}<x<n
$$

we have

$$
e^{x} \leq U(n, x)
$$

with equality if and only if $x=0$. Moreover, for $0 \leq x<1$ and $1 \leq n \leq 2$ we have

$$
e^{x} \leq U(n, x) \leq \frac{1}{1-x},
$$

Received by the editors January 15, 2005 and, in revised form, July 5, 2005 and August 5, 2005.

2000 Mathematics Subject Classification. Primary 33B10; Secondary 11A99.

Key words and phrases. Algebraic bounds, exponential function, polynomials.

This study was supported (in part) by research funds from Chosun University, 2004.

(C)2006 American Mathematical Society 
and for $x<0$ and $0<n \leq 1$ we have

$$
e^{x} \leq \frac{1}{1-x} \leq U(n, x)
$$

Here $U(n, x)$ is smoothly and densely algebraic in $n$ in the sense that it is an algebraic function of $x$ whenever $n$ is rational and this algebraic function changes by arbitrarily small amounts on compact sets for sufficiently small rational changes in $n$.

Another upper bound for $e^{x}$ that generalizes (1.1) is Karamata's [2]

$$
e^{x} \leq \sum_{k=0}^{n-1} \frac{x^{k}}{k !}+\frac{x^{n}}{n !} \frac{n}{n-x},
$$

provided $n$ is a positive integer and $0 \leq x<n$. This is tighter for $0<x<n$ but often fails for $x<0$. Also, since $n-1$ is the upper limit of the summation here, it is not smoothly algebraic in $n$. Another tighter bound for $e^{x}$ is Sewell's [4]

$$
e^{x} \leq\left(1+\frac{x}{n}\right)^{n+(x / 2)}
$$

for $n$ a positive integer and $x \geq 0$. This is not algebraic in $x$, and can fail for $x<0$.

The change of variable given by replacing $x$ with

$$
\frac{n(x-1)}{n+x-1}
$$

plays an important role here. In fact, it is immediate that the first part of Theorem 1.1 is equivalent to

Theorem 1.2. For real $n \geq 1$ and $x>0$ we have

$$
\exp \left(\frac{n(x-1)}{n+x-1}\right) \leq \frac{n-1+x^{n}}{n}
$$

with equality if and only if $x=1$.

Our proof for Theorem 1.2 will be in the spirit of $\S 2.15$ of [1] where some "fundamental inequalities" that also lead to the inequality between the arithmetic and geometric means, including

$$
x^{r}-1>r(x-1), \quad r>1, \quad x>0, \quad x \neq 1,
$$

are proved for all real $r>1$ by first establishing them for rational numbers and then taking limits. Also the proof of Theorem 1.2 will rely on the polynomial

$$
K(p, q, x):=q^{2}\left(x^{p+q}-x^{p}\right)+q(p-q)\left(x^{p}-x^{p-q}\right)+p(p-q)\left(x^{p-q}-1\right),
$$

where both $p$ and $q$ are integers and $p>q$. For our convenience, we write $K(x)=$ $K(p, q, x)$.

For a survey of rational bounds for $e^{x}$ see pp. 266-270 of 3 . The examination of the inequalities between $(1-x)^{-1}$ and $U(n, x)$ from the point of view of their power series expansions leads to questions about a certain sequence of polynomials; see $\S 3$. We remark here that for $0 \leq x<1$ the power series of $U(n, x)-(1-x)^{-1}$ about $x=0$ is

$$
\frac{(n-1)(n-2)}{2 n} x^{2}+\frac{1}{6}\left(2+\frac{6}{n^{2}}-\frac{6}{n}-3 n+n^{2}\right) x^{3}+\cdots .
$$


So for $n>2$ and small $x>0$ we have $U(n, x)>(1-x)^{-1}$. For $0 \leq x<1$ and $n$ large some simple asymptotics (details omitted) show that $(1-x)^{-1}<U(n, x)$ for $x \leq 1-c^{n}$ for a fixed $c>1 / 2$, while $U(n, x)<(1-x)^{-1}$ for $x \geq 1-c^{n}$ for a fixed $c \leq 1 / 2$.

\section{Proofs}

We begin with a lemma that leads to the inequalities between $(1-x)^{-1}$ and $U(n, x)$, and then proceed to the inequalities between $e^{x}$ and $U(n, x)$. This latter inequality is of course immediate when $x<1$ and $(1-x)^{-1} \leq U(n, x)$.

Lemma 2.1. (a) Let $c=1-1 / n$, where $1 \leq n \leq 2$ and $0 \leq x<1$. Then

$$
l_{1}:=\frac{n c}{1+c x}+\frac{1}{1-\frac{x}{n}} \leq \frac{n c}{1+n c x}+\frac{1}{1-x}=: r_{1} .
$$

(b) Let $0<a \leq b, 1 \leq b$, and $x \geq 0$. Then

$$
l_{2}:=\frac{a}{1+\frac{a}{b} x}+\frac{b}{1+b x} \leq \frac{a}{1+a x}+\frac{b}{1+x}=: r_{2} .
$$

Proof. For (a) we have

$$
r_{1}-l_{1}=\frac{(n-1)(1+c n) x(2-n+c x(1+n))}{(x-1)(x-n)(1+c x)(1+c n x)} \geq 0,
$$

while for (b) we have

$$
r_{2}-l_{2}=\frac{(b-1)(b-a) x(a+b+a(1+b) x)}{(1+x)(1+a x)(b+a x)(b x+1)} \geq 0 .
$$

We now proceed to the right side of the second part of Theorem 1.1. Observe that

$$
n \log (1+c x)-n \log \left(1-\frac{x}{n}\right) \leq \log (1+n c x)-\log (1-x)
$$

since there is equality when $x=0$, and the corresponding inequality between the derivatives of each side follows from (a) of Lemma 2.1. Hence

$$
\left(\frac{1+c x}{1-\frac{x}{n}}\right)^{n} \leq \frac{1+n c x}{1-x}
$$

and we obtain $U(n, x) \leq 1 /(1-x)$. For the right side of the third part of Theorem 1.1 a similar argument using (b) of Lemma 2.1 yields

$$
\left(\frac{1+\frac{a}{b} x}{1+x}\right)^{b} \leq \frac{1+a x}{1+b x}
$$

Here we may take $b=\frac{1}{n}$ and $a=\frac{1}{n}-1$ for $0<n \leq 1$, so

$$
\frac{1-(n-1) x}{1+x} \leq\left(\frac{1-\left(1-\frac{1}{n}\right) x}{1+\frac{x}{n}}\right)^{n} .
$$

Upon replacing $x$ by $-x$ (so that $x \leq 0$ ), we obtain

$$
\frac{1}{1-x} \leq U(n, x) \text {. }
$$


For the proof of Theorem 1.2 (and hence of the remaining first part of Theorem 1.1) we observe that (1.2) is equivalent to

$$
g(x):=\frac{n(x-1)}{n+x-1} \leq \log \left(\frac{n-1+x^{n}}{n}\right)=: f(x) .
$$

Since both sides of (2.1) are zero when $x=1$ we may apply the following lemma (proof omitted) to reduce it to an inequality not involving transcendental functions.

Lemma 2.2. Let $f(x)$ and $g(x)$ be differentiable functions on a finite or infinite interval I containing 1 such that $f(1)=g(1)$, and such that $g^{\prime}(x) \geq f^{\prime}(x)$ for $x<1$ and $g^{\prime}(x) \leq f^{\prime}(x)$ for $x>1$. Then $g(x) \leq f(x)$.

Now

$$
g^{\prime}(x)=\frac{n^{2}}{(n-1+x)^{2}} \quad \text { and } \quad f^{\prime}(x)=\frac{n x^{n-1}}{n-1+x^{n}} .
$$

Replace $n$ by $p / q$ where both $p$ and $q$ are positive integers, $p>q$. The change of variable $x$ by $x^{q}$ takes 1 to 1 and $(0, \infty)$ to $(0, \infty)$. To verify the hypothesis of Lemma 2.2 we need to show that $H(x)$ has the same sign as $(x-1)$, where

$$
\begin{aligned}
H(x) & :=H(p, q, x):=f^{\prime}(x)-g^{\prime}(x) \\
& =\frac{p x^{p-q}}{p+q\left(x^{p}-1\right)}-\frac{p^{2}}{\left(p+q\left(x^{q}-1\right)\right)^{2}} \\
& =\frac{p K(x)}{\left(p-q+q x^{p}\right)\left(p-q+q x^{q}\right)^{2}}
\end{aligned}
$$

and

$$
K(x)=q^{2}\left(x^{p+q}-x^{p}\right)+q(p-q)\left(x^{p}-x^{p-q}\right)+p(p-q)\left(x^{p-q}-1\right) .
$$

Using the identity

$$
x^{s}-x^{t}=\left(\frac{x^{s}-1}{x-1}-\frac{x^{t}-1}{x-1}\right)(x-1)
$$

for $s \geq t \geq 0$ and the expansion of the terms in (2.2) into geometric series, we see that $K(x)$ is the product of $x-1$ with polynomials in $x$, all of whose coefficients are nonnegative. Hence $K(x)$ has the same sign as $x-1$. The inequality of the theorem for rational $n$ now follows from Lemma 2.2. For real $n \geq 1$, it follows by letting $p / q \rightarrow n$ where $p$ and $q$ run through sequences of integers such that $p>q \geq 1$. The strict inequality for $x \neq 1$ follows from the fact that the functions in $x$

$$
\exp \left(\frac{n(x-1)}{n+x-1}\right) \text { and } \frac{n-1+x^{n}}{n}
$$

are strictly increasing for $x>0$.

\section{A SEQUence of POLynomials}

It is possible that the $U(n, x) \leq(1-x)^{-1}$ inequality for $1 \leq n \leq 2$ can be strengthened to an inequality between the corresponding power series coefficients. In fact, we can make a stronger conjecture. Write

$$
\frac{1}{n-1}\left(U(n, x)-(1-x)^{-1}\right)=\sum_{k=2}^{\infty} \frac{P_{k}(n) x^{k}}{k ! n^{k-1}} .
$$


Here $P_{2}(n)=n-2, P_{3}(n)=n^{3}-2 n^{2}-6$ and

$$
P_{4}(n)=n^{5}-5 n^{4}+18 n^{3}-48 n^{2}+12 n-24 .
$$

The unique real zeros of $P_{2}, P_{3}$ and $P_{4}$ are $n=2, n=2.7776 \cdots$ and $n=3.5934 \cdots$, respectively. We conjecture that each $P_{k}(n)$ for $k \geq 4$ is a monic polynomial of degree $2 k-3$ whose coefficients alternate in sign, and has a unique real root $r_{k}$ that exceeds the real part of every other root of $P_{k}(n)$. Moreover,

$$
0<r_{k+1}-r_{k}<1 \quad \text { and } \quad \lim _{k \rightarrow \infty}\left(r_{k+1}-r_{k}\right)=1 .
$$

It also seems that $k$ ! divides $P_{k}(2)$.

To describe qualitatively the conjectural zero distribution of $P_{k}(n)$ we employ polar coordinates $r$ and $\phi$ to describe a certain curve $\theta$. It is the cardioid $H$ given by $r=1+\cos \phi$ together with that part of the circle $C$ defined by $r=1 / 4$ that lies outside of $H$. The left vertical tangent to $C$, call it $T$, is tangent to the cardioid at two points, and (much more crudely) the $\theta$-curve is topologically equivalent to the Greek letter $\theta$. Note that the cusp of $H$ is inside of the circle $C$. The conjecture is that the zeros of $P_{k}(n)$ for $k$ large lie on or very close to a curve similar (in the non-technical sense) to $\theta$ that has the imaginary axis as a line of triple tangency analogous to $T$. Also, about $1 / 3$ of the zeros lie on or inside the part of the $\theta$-curve that corresponds to the union of its $C$ part with that part of $H$ that lies inside of the full circle $C$.

\section{ACKNOWLEDGMENT}

The author expresses his thanks to Prof. Kenneth B. Stolarsky of the University of Illinois at Urbana-Champaign, USA, for helpful conversations. The author is grateful to the referee of this paper for useful comments and suggestions that led to further development of an earlier version.

\section{REFERENCES}

[1] G. H. Hardy, J. E. Littlewood, G. Pólya, Inequalities, Cambridge, 1975. MR0944909 (89d:26016)

[2] J. Karamata, Sur l'approximation de $e^{x}$ par des fonctions rationnelles (in Serbian), Bull. Soc. Math. Phys. Serbie 1 (1949), 7-19. MR0031124(11:104a)

[3] D. S. Mitrinovic, Analytic Inequalities, Springer-Verlag, New York, 1970. MR0274686 (43:448)

[4] W. E. Sewell, Some inequalities connected with exponential function (in Spanish), Rev. Ci (Lima) 40 (1938), 453-456.

[5] J. E. Wetzel, On the functional inequality $f(x+y) \geq f(x) f(y)$, Amer. Math. Monthly 74 (1967), 1065-1068. MR0228865 (37:4444)

Department of Mathematics, College of Natural Science, Chosun University, 375 Susuk-DOng, Dong-Gu, Gwanguu, 501-759 Korea

E-mail address: shkim17@mail.chosun.ac.kr 